\title{
Preclinical Investigation of Inhibition of the DNA Damage Response as a Targetted Therapy in Myeloproliferative Neoplasms Shows Synergism of ATR Inhibitors with Standard-of-Care Treatment ${ }^{\dagger}$
}

\author{
Aleksander Ślusarczyk ${ }^{1, \ddagger(\mathbb{D}) \text {, Helen E. Bryant }}{ }^{1}\left(\mathbb{D}\right.$, Edwin Chen $^{2}$, Ian Hitchcock ${ }^{3} \mathbb{D}$, Martin Zeidler $^{4}$, \\ Andrew Chantry ${ }^{1,5}$ and Sally Thomas ${ }^{1,5, *}$
}

1 Department of Oncology and Metabolism, University of Sheffield Medical School, Sheffield S10 2RX, UK; slusarczyk.aleksander@gmail.com (A.Ś.); h.bryant@sheffield.ac.uk (H.E.B.); a.d.chantry@sheffield.ac.uk (A.C.)

2 Department of Life Sciences, University of Westminster, London W1B 2HW, UK; E.Chen@westminster.ac.uk

3 York Biomedical Research Institute, Department of Biology, University of York, York YO10 5DD, UK; ian.hitchcock@york.ac.uk

4 The Bateson Centre, Department of Biomedical Science, University of Sheffield, Sheffield S10 2TN, UK; m.zeidler@sheffield.ac.uk

5 Department of Haematology, Sheffield Teaching Hospitals NHS Foundation Trust, Sheffield S10 2JF, UK

* Correspondence: sally.j.thomas@sheffield.ac.uk

+ Presented at the 1st International Electronic Conference on Cancers: Exploiting Cancer Vulnerability by Targeting the DNA Damage Response, 1-14 February 2021; Available online: https:/ /iecc2021.sciforum.net/.

check for

updates

Citation: Ślusarczyk, A.; Bryant, H.E.; Chen, E.; Hitchcock, I.; Zeidler, M.; Chantry, A.; Thomas, S. Preclinical Investigation of Inhibition of the DNA Damage Response as a Targetted Therapy in Myeloproliferative Neoplasms Shows Synergism of ATR Inhibitors with Standard-of-Care Treatment. Med. Sci. Forum 2021, 3, 17. https: / /doi.org/10.3390/ IECC2021-09209

Academic Editor: Nicola Curtin

Published: 29 January 2021

Publisher's Note: MDPI stays neutral with regard to jurisdictional claims in published maps and institutional affiliations.

Copyright: (c) 2021 by the authors. Licensee MDPI, Basel, Switzerland. This article is an open access article distributed under the terms and conditions of the Creative Commons Attribution (CC BY) license (https:// creativecommons.org/licenses/by/ $4.0 /)$. $\ddagger$ Current address: Department of Immunology, Medical University of Warsaw, 02-097 Warsaw, Poland.

Abstract: Myeloproliferative neoplasms (MPNs) are a group of haematological malignancies arising from haematopoietic stem cells (HSCs) with acquired driver mutations in JAK2, MPL and CALR. Current therapies are not selective for the mutant HSC population. Increased replication stress is seen in the presence of mutant $J A K 2$, suggesting DNA damage response inhibitors (DDRi) may differentially affect mutant HSCs over wild-type HSCs to restore normal haematopoiesis. Using JAK2V617F and CALR (del 52) mutant cell lines, we observed that ATR inhibition (ATRi) by AZD6738 and VE-821 significantly reduced viability. The combination of ATRi and a hydroxyurea/a JAK1/2 inhibitor-ruxolitinib—demonstrated high synergism in both apoptosis induction and proliferation arrest. This study provides preliminary evidence that ATRi combined with standard therapies may be exploited in MPNs harbouring JAK2 and CALR mutations.

Keywords: myeloproliferative disorders; Janus kinase 2; calreticulin; ataxia telangiectasia mutated proteins; Janus kinase inhibitors; hydroxyurea

\section{Introduction}

Myeloproliferative neoplasms (MPNs) are a group of haematological malignancies that arise from haematopoietic stem cells with acquired driver mutations in $J A K 2, M P L$ and CALR. MPNs comprise polycythaemia vera (PV), essential thrombocythemia (ET) and myelofibrosis (MF), which are characterised by aberrant blood cell production resulting in thrombosis, splenomegaly, bone marrow fibrosis and leukaemia [1].

Interestingly, genes involved in the DNA double-strand break (DSB) repair pathways of BRCA-dependent homologous recombination repair (HRR) and DNA-dependent protein kinase-mediated nonhomologous end-joining (D-NHEJ) were upregulated in the presence of JAK2(V617F), MPL(W515L) and CALR(del52), which are frequently observed in MPNs [2]. As JAK2 activation promotes expression of HRR and D-NHEJ genes, it might prevent DNA damage and contribute to chemotherapy resistance [2]. As previously reported, the JAK1/2 inhibitor ruxolitinib caused downregulation of key members of HRR 
(BRCA1, RAD51) and D-NHEJ (LIG4) in JAK2(V617F), MPL(ex10mut) and CALR(del52)+ cell lines, resulting in reduced HRR and D-NHEJ activities [2].

The currently used MPN therapies include hydroxyurea, which induces DNA doublestrand breaks (DSB) [3], and ruxolitinib. They are not selective for the disease clone, do not induce complete remission and are limited by frequent side effects. In particular, hydroxyurea therapy is often associated with adverse reactions, such as anaemia, leukopenia, gastrointestinal symptoms and mucocutaneous toxicity [4]. Hydroxyurea resistance or intolerance occurs in 15 to $24 \%$ of patients with PV [4,5]. Ruxolitinib has been shown to prolong life in patients with myelofibrosis [6] but revealed limited benefit in PV [7] and ET [8], leading to its registration as second-line therapy. All this highlights the need for the exploration of new regimens and provides the rationale for the evaluation of combination therapies.

DNA damage repair system targeting compounds have been little investigated in haematological malignancies. Tumour reliance on more than one DDR pathway leads to ineffective cell death induction upon single-agent use [9]. This provides a rationale to evaluate DDRi in combinations with drugs that may contribute to DSB formation (hydroxyurea) or indirect DDR pathway inhibition (ruxolitinib). Using JAK2V617F and CALR (del 52) mutant cell lines, we aimed to determine the effect of single-agent DDRi on cell viability and apoptosis. Moreover, we evaluated the efficacy of DDRi in combination with currently registered therapies-hydroxyurea and ruxolitinib.

\section{Materials and Methods}

\subsection{Cell Culture}

Cell lines expressing JAK2 (V617F)-HEL and CALR (del52)-MARIMO were seeded at $0.5 \times 10^{6}$ cells $/ \mathrm{mL}$ in RPMI 1640 + GlutaMAX medium supplemented with $10 \%$ FBS, 100 units $/ \mathrm{mL}$ penicillin and $100 \mu \mathrm{g} / \mathrm{mL}$ streptomycin. All cells were cultured in a $37^{\circ} \mathrm{C}$ humidified incubator with $5 \% \mathrm{CO}_{2}$.

\subsection{Drugs}

Both cell lines were treated with a drug panel comprising hydroxyurea, ruxolitinib, methotrexate, AZD6738 (ATRi), NU7441 (DNA-PKi), Olaparib (PARPi) and VE-821 (ATRi). All drugs were dissolved in 100\% DMSO. The maximum concentration of DMSO in treated cells was $0.3 \%$ and a DMSO alone control of equal concentration was consistently used. Concentrations used for the experiments were designed based on other studies investigating DDRi [9-12].

\subsection{Viability and Proliferation Assessment}

Cells at a density of $2 \times 10^{5} / \mathrm{mL}$ were exposed to single drugs and their combinations. After $48 \mathrm{~h}$ exposure, proliferation assessment and apoptosis assay was performed. AlamarBlue Cell Viability Reagent (Thermofisher) was used for cell proliferation evaluation according to the manufacturer's instruction. AlamarBlue was added to a final dilution of 1:10. The colour was developed after $4 \mathrm{~h}$ of incubation, and measurements were performed on a SoftMax Pro 5.2 plate reader at an excitation wavelength of $570 \mathrm{~nm}$ and emission wavelength of $600 \mathrm{~nm}$. Cell viability was calculated as the ratio of absorbance of treated cells to the absorbance of control cells. A Dead Cell Apoptosis Kit (Thermofisher) was used for apoptosis assessment on a BD FACSCalibur, with cells gated on forward and side scatter and 10,000 events recorded per condition. Staining with annexin V-FITC and propidium iodide for flow cytometry was carried out to evaluate the cell death mechanism.

\subsection{Statistical Analysis}

Statistical analysis was performed using GraphpadPrism v8.4.3. Data are represented as mean and standard deviation. The combination index and synergism were evaluated in CompuSyn software. Synergism was defined as a combination index (CI) lower than $1.0[13]$. 


\section{Results}

\subsection{Single Drugs}

In JAK2- and CALR-mutated cell lines, ATR inhibition by AZD6738 or VE-821, DNAPK inhibition by NU7441 and hydroxyurea each reduced viability compared to DMSO control, whereas PARP inhibition by olaparib had a minimal effect. Ruxolitinib alone had a modest effect on the presence of JAK2V617F and no effect on CALR (del 52)-mutated cells (Figure 1).

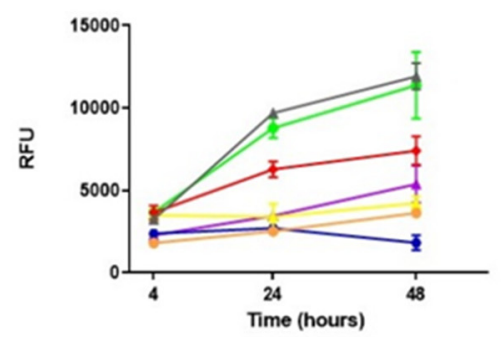

(a)

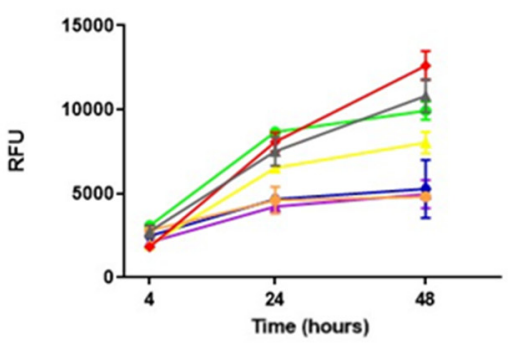

- DMSO 0.3

$\rightarrow$ Ruxolitinib $1 \mu \mathrm{M}$

- Hydroxyurea $30 \mu \mathrm{g} / \mathrm{ml}$

- Olaparib $5 \mu \mathrm{M}$

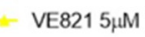

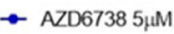

- NU7441 5 $4 \mathrm{M}$

Figure 1. Effect of standard therapies and DDRi on cell viability and proliferation, assessed with AlamarBlue assays, in cell lines representing MPNs. Relative fluorescence unit (RFU) at different time points for (a) HEL cells (JAK2V617F); (b) MARIMO cells (CALR del 52). Error bars show mean and standard deviation of three replicates.

\subsection{Drug Combinations}

Combinations of DDRi with currently registered drugs-ruxolitinib and hydroxyurearevealed promising synergistic toxicity. The combination of both different ATRi (AZD6738/ VE-821) and hydroxyurea demonstrated consistent high synergism in both cell lines in terms of cell viability reduction (Figure $2 \mathrm{a}, \mathrm{b}$ ). For the combination of $20 \mu \mathrm{g} / \mathrm{mL}$ hydroxyurea and $1 \mu \mathrm{M}$ AZD6738, the CI was 0.2 in HEL cells and 0.1 in MARIMO cells. Synergistic toxicity was also observed for the ruxolitinib and ATR inhibitor combination but only in the JAK2-mutated cell line, where the $\mathrm{CI}$ was 0.3 for the combination of $1 \mu \mathrm{M}$ ruxolitinib and $2 \mu \mathrm{M}$ AZD6738 (Figure 2c).

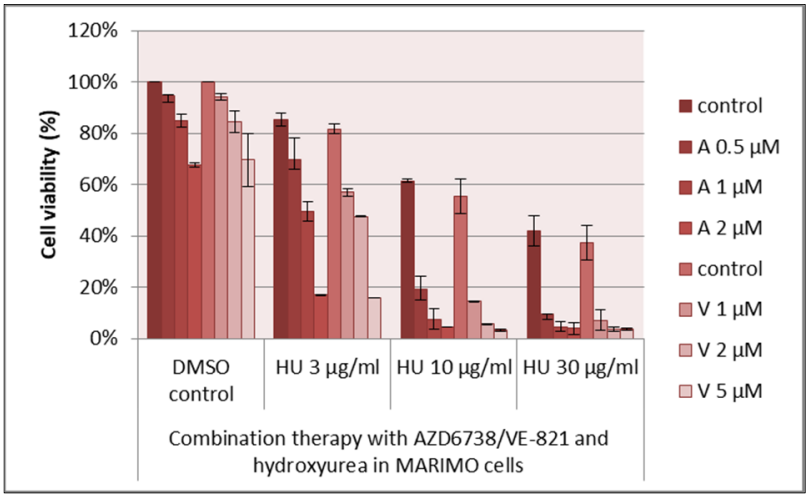

(a)

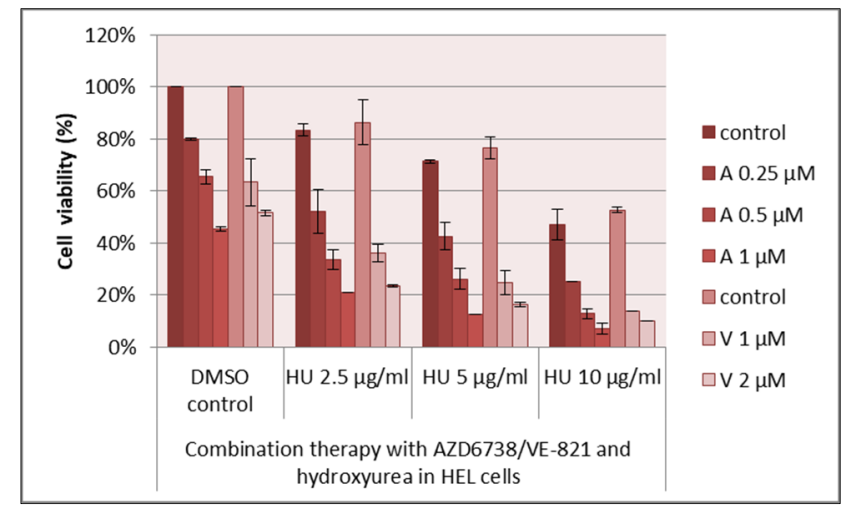

(b)

Figure 2. Cont. 


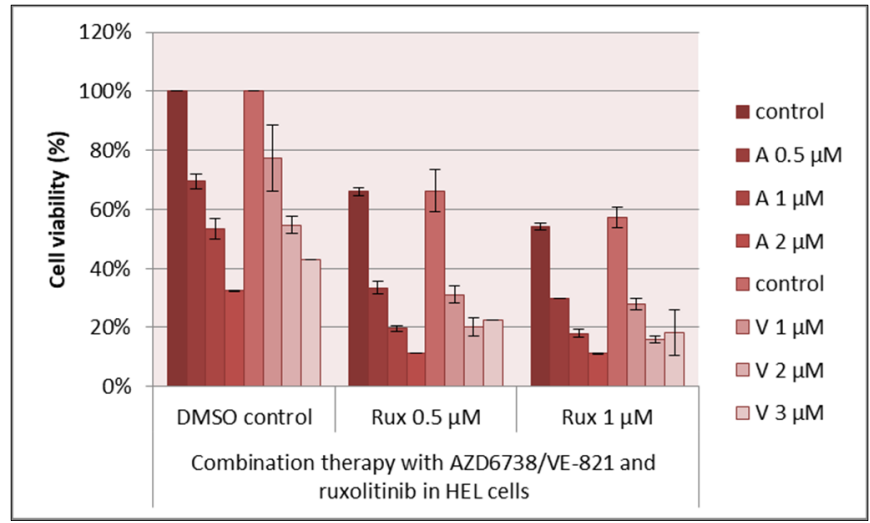

(c)

Figure 2. Cell viability in MARIMO cells (a) and HELcells (b) measured by AlamarBlue assay after 48 h exposure to combinations of hydroxyurea (HU) with ATRi- AZD6738 (A) or VE-821 (V), and in HEL cells (c) after exposure to ruxolitinib (Rux) with ATRi. Error bars show mean and standard deviation of two replicates.

\subsection{Apoptosis}

The effects seen on AlamarBlue assays could be a consequence of decreased cell proliferation or increased cell death. To investigate these alternatives, the percentage of live cells following drug treatment was measured for MARIMO cells using flow cytometry and annexinV-FITC and propidium iodide staining. The percentage of live cells following $0.1 \%$ DMSO treatment was $94 \%$ (Figure $3 a$ ) and was minimally affected by single-agent hydroxyurea $10 \mu \mathrm{g} / \mathrm{mL}$ (83\%) (Figure 3b), AZD6738 $2 \mu \mathrm{M}$ (92\%) (Figure 3c) or VE-821 $5 \mu \mathrm{M}$ (91\%) (Figure 3e). Combining hydroxyurea and ATRi produced a substantial reduction in the percentage of live cells, to $19 \%$ for $10 \mu \mathrm{g} / \mathrm{mL}$ hydroxyurea with $2 \mu \mathrm{M}$ AZD6738 (Figure 3d) and $24 \%$ for $10 \mu \mathrm{g} / \mathrm{mL}$ hydroxyurea with $5 \mu \mathrm{M}$ VE-821 (Figure 3f).

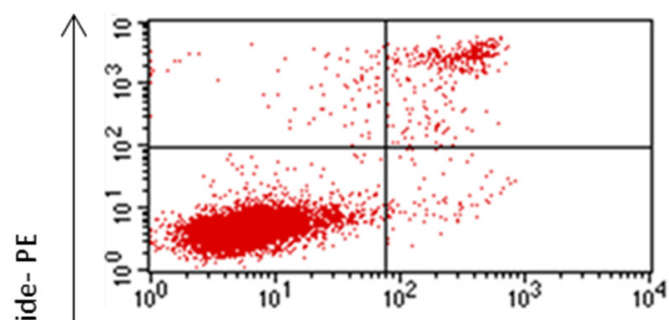

(a)

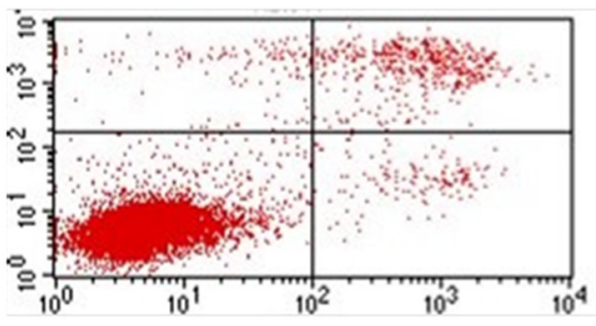

(c)

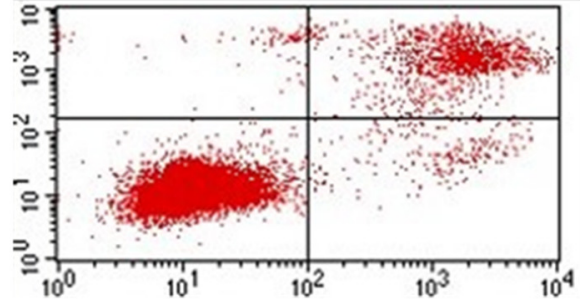

(b)

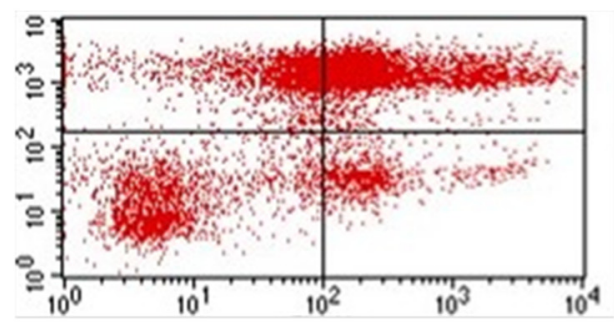

(d)

Annexin V-FITC

Figure 3. Cont. 


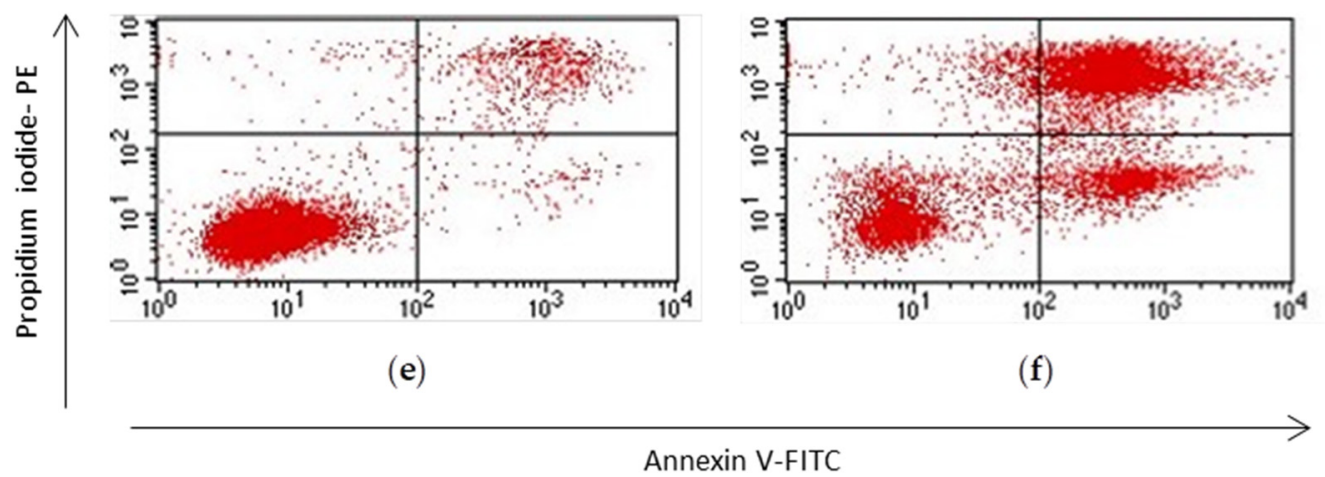

Figure 3. The combination of hydroxyurea and ATRi leads to a substantial synergistic increase in the apoptosis induction in MARIMO cells after $48 \mathrm{~h}$ exposure. (a) DMSO 0.1\% control, (b) $10 \mu \mathrm{g} / \mathrm{mL}$ hydroxyurea, (c) $2 \mu \mathrm{M}$ AZD6738, (d) combination of $2 \mu \mathrm{M}$ AZD6738 and $10 \mu \mathrm{g} / \mathrm{mL}$ hydroxyurea, (e) $5 \mu \mathrm{M}$ VE-821, (f) combination of $5 \mu \mathrm{M}$ VE-821 and $10 \mu \mathrm{g} / \mathrm{mL}$ hydroxyurea; only one experiment was performed; $x$ axis_annexin V-FITC, $y$ axis-propidium iodide.

\section{Discussion}

Investigations into DDRi pave the way for new therapies in haematological malignancies [14]. In this study, we evaluated our hypothesis that DDRi might target leukemic cells in the presence of mutated JAK2 and CALR and that it might enhance the efficacy of currently used drugs. ATRi AZD6738 and VE-821 showed effects as single agents. ATRi demonstrated a synergistic effect in combination with currently approved drugs-hydroxyurea and ruxolitinib. Synergism between ATRi and chemotherapeutics has previously been investigated in AML cell lines and solid tumours [10,15]. Interestingly, AZD6738 was previously evaluated in chronic lymphocytic leukaemia (CLL) in vitro and in vivo studies, which reported high efficacy in p53-null or ATM-null CLL cells but not in wild-type cells [9].

The synergistic toxicity of the combination of hydroxyurea and ATRi observed in our study can be explained by the genotoxic effect of both chemotherapeutic and ATRi. hydroxyurea targets ribonucleotide reductase, consequently, it promotes chromosome fragility and DNA DSBs [16]. As ATRi leads to impairment of DNA single- and doublestand break repair, accumulation of breaks consequently causes apoptosis. Moreover, combination therapies including ATRi and hydroxyurea might be of clinical value, as hydroxyurea resistance/intolerance occurs relatively frequently [5].

Olaparib was ineffective when used in monotherapy. However, the combination of ruxolitinib and olaparib has previously been demonstrated to synergistically affect primary MPN cells harbouring JAK2 or CALR mutations [2]. Finally, ruxolitinib treatment showed efficacy only in cells with JAK2V617F, not in CALR-mutated ones. Consequently, its synergism with ATRi was revealed only in the presence of JAK2 mutation.

\section{Conclusions}

To conclude, DDRi reduces viability in cells expressing the driver mutations seen in MPNs. ATRi constitutes a promising therapeutic approach in haematological malignancies characterised by increased reliance on DNA damage repair pathways activity. Most notably, ATRi exhibits a synergistic effect with the current standard-of-care treatment, hydroxyurea and ruxolitinib, to reduce cell viability by inducing apoptosis. This study provides preliminary evidence that ATRi combined with standard therapies may be exploited in MPNs harbouring JAK2 and CALR mutations. Further preclinical investigation is warranted.

Author Contributions: Conceptualisation, A.Ś., S.T., H.E.B., A.C., M.Z., I.H. and E.C.; methodology, A.Ś., S.T., A.C. and H.E.B.; formal analysis, A.Ś. and S.T.; investigation, A.Ś.; resources, S.T., H.E.B., A.C., M.Z. and I.H.; data curation, A.S. and S.T.; writing—original draft preparation, A.S.; writingreview and editing, S.T., H.E.B., A.C., M.Z., I.H. and E.C.; visualisation, A.Ś. and S.T.; supervision, 
S.T., A.C., H.E.B. and M.Z.; project administration, S.T.; funding acquisition, S.T., A.C., H.E.B., I.H. and M.Z. All authors have read and agreed to the published version of the manuscript.

Funding: This research was funded by the White Rose University Consortium Collaboration Fund and supported by the National Institute for Health Research.

Institutional Review Board Statement: Not applicable.

Informed Consent Statement: Not applicable.

Data Availability Statement: The data presented in this study are available on request from the corresponding author.

Acknowledgments: Thank you to S. Clarke at the University of Sheffield Flow Cytometry core facility technical support for the flow cytometry experiments. Thank you to N. Tawfiq, S. Lawson, J. Down, G. Stewart and M. A. Garcia for help with experimental techniques. MARIMO cells were a kind gift from Professor Hitoshi Kiyoi of Nagoya University via Stuart Scott of UKNEQUAS leucocyte Immunophenotyping.

Conflicts of Interest: The authors declare no conflict of interest.

\section{References}

1. Vainchenker, W.; Kralovics, R. Genetic basis and molecular pathophysiology of classical myeloproliferative neoplasms. Blood 2017, 129, 667-679. [CrossRef] [PubMed]

2. Nieborowska-Skorska, M.; Maifrede, S.; Dasgupta, Y.; Sullivan, K.; Flis, S.; Le, B.V.; Solecka, M.; Belyaeva, E.A.; Kubovcakova, L.; Nawrocki, M.; et al. Ruxolitinib-induced defects in DNA repair cause sensitivity to PARP inhibitors in myeloproliferative neoplasms. Blood 2017, 130, 2848-2859. [CrossRef] [PubMed]

3. Petermann, E.; Orta, M.L.; Issaeva, N.; Schultz, N.; Helleday, T. Hydroxyurea-stalled replication forks become progressively inactivated and require two different RAD51-mediated pathways for restart and repair. Mol. Cell 2010, 37, 492-502. [CrossRef] [PubMed]

4. Demuynck, T.; Verhoef, G.; Delforge, M.; Vandenberghe, P.; Devos, T. Polycythemia vera and hydroxyurea resistance/intolerance: A monocentric retrospective analysis. Ann. Hematol. 2019, 98, 1421-1426. [CrossRef] [PubMed]

5. Barosi, G.; Besses, C.; Birgegard, G.; Briere, J.; Cervantes, F.; Finazzi, G.; Gisslinger, H.; Griesshammer, M.; Gugliotta, L.; Harrison, C.; et al. A unified definition of clinical resistance/intolerance to hydroxyurea in essential thrombocythemia: Results of a consensus process by an international working group. Leukemia 2007, 21, 277-280. [CrossRef] [PubMed]

6. Cervantes, F.; Vannucchi, A.M.; Kiladjian, J.J.; Al-Ali, H.K.; Sirulnik, A.; Stalbovskaya, V.; McQuitty, M.; Hunter, D.S.; Levy, R.S.; Passamonti, F.; et al. Three-year efficacy, safety, and survival findings from COMFORT-II, a phase 3 study comparing ruxolitinib with best available therapy for myelofibrosis. Blood 2013, 122, 4047-4053. [CrossRef] [PubMed]

7. Kiladjian, J.J.; Zachee, P.; Hino, M.; Pane, F.; Masszi, T.; Harrison, C.N.; Mesa, R.; Miller, C.B.; Passamonti, F.; Durrant, S.; et al. Long-term efficacy and safety of ruxolitinib versus best available therapy in polycythaemia vera (RESPONSE): 5-year follow up of a phase 3 study. Lancet Haematol. 2020, 7, e226-e237. [CrossRef]

8. Harrison, C.N.; Mead, A.J.; Panchal, A.; Fox, S.; Yap, C.; Gbandi, E.; Houlton, A.; Alimam, S.; Ewing, J.; Wood, M.; et al. Ruxolitinib vs best available therapy for ET intolerant or resistant to hydroxycarbamide. Blood 2017, 130, 1889-1897. [CrossRef] [PubMed]

9. Kwok, M.; Davies, N.; Agathanggelou, A.; Smith, E.; Petermann, E.; Yates, E.; Brown, J.; Lau, A.; Stankovic, T. Synthetic lethality in chronic lymphocytic leukaemia with DNA damage response defects by targeting the ATR pathway. Lancet 2015, 385 (Suppl. 1), S58. [CrossRef]

10. Vendetti, F.P.; Lau, A.; Schamus, S.; Conrads, T.P.; O'Connor, M.J.; Bakkenist, C.J. The orally active and bioavailable ATR kinase inhibitor AZD6738 potentiates the anti-tumor effects of cisplatin to resolve ATM-deficient non-small cell lung cancer in vivo. Oncotarget 2015, 6, 44289-44305. [CrossRef] [PubMed]

11. Chaudhuri, L.; Vincelette, N.D.; Koh, B.D.; Naylor, R.M.; Flatten, K.S.; Peterson, K.L.; McNally, A.; Gojo, I.; Karp, J.E.; Mesa, R.A.; et al. CHK1 and WEE1 inhibition combine synergistically to enhance therapeutic efficacy in acute myeloid leukemia ex vivo. Haematologica 2014, 99, 688-696. [CrossRef] [PubMed]

12. Alikarami, F.; Safa, M.; Faranoush, M.; Hayat, P.; Kazemi, A. Inhibition of DNA-PK enhances chemosensitivity of B-cell precursor acute lymphoblastic leukemia cells to doxorubicin. Biomed. Pharmacother. Biomed. Pharmacother. 2017, 94, 1077-1093. [CrossRef] [PubMed]

13. Chou, T.C. Theoretical basis, experimental design, and computerized simulation of synergism and antagonism in drug combination studies. Pharmacol. Rev. 2006, 58, 621-681. [CrossRef] [PubMed]

14. Alhmoud, J.F.; Mustafa, A.G.; Malki, M.I. Targeting DNA Repair Pathways in Hematological Malignancies. Int. J. Mol. Sci. 2020, 21, 7365. [CrossRef] [PubMed] 
15. Ma, J.; Li, X.; Su, Y.; Zhao, J.; Luedtke, D.A.; Epshteyn, V.; Edwards, H.; Wang, G.; Wang, Z.; Chu, R.; et al. Mechanisms responsible for the synergistic antileukemic interactions between ATR inhibition and cytarabine in acute myeloid leukemia cells. Sci. Rep. 2017, 7, 41950. [CrossRef] [PubMed]

16. Hoffman, E.A.; McCulley, A.; Haarer, B.; Arnak, R.; Feng, W. Break-seq reveals hydroxyurea-induced chromosome fragility as a result of unscheduled conflict between DNA replication and transcription. Genome Res. 2015, 25, 402-412. [CrossRef] [PubMed] 